\title{
Implementation of ECG QRS complex detector for Body Sensor Networks
}

\author{
Bhavani .V, P.Aparna, K.Praveena \\ Assistant professor, Department of ECE, M.V.J.College of Engineering, Bangalore, Karnataka.
}

\begin{abstract}
This paper aims to present a mathematical morphological method to remove baseline wandering and background noise is removed from original ECG signal. Then the multipixel modulus accumulation is employed to act as a low-pass filter to enhance the QRS complex and improve the signal-to-noise ratio. The performance of the algorithm is evaluated with standard MIT-BIH arrhythmia database. Corresponding power and area efficient VLSI architecture is designed and implemented on a commercial nano-FPGA. High detection rate and high speed demonstrate the effectiveness of the proposed detector.

Index Terms: Body sensor networks (BSNs), electrocardiogram (ECG) sensor, field-programmable gate array (FPGA), mathematical morphology, QRS detection, very-large-scale integration (VLSI) architecture
\end{abstract}

\section{Introduction}

The last decade has witnessed a rapid surge of interest in new sensing and monitoring devices for healthcare and the use of wearable/wireless devices and sensor networks for clinical applications.

As one of the important physiological sensor nodes in BSN, wearable electrocardiogram (ECG) sensor is dedicated to measuring the rate and regularity of heartbeats as well as the size and position of the chambers, the presence of any damage to the heart and the effects of drugs or devices used to regulate

the heart [1]. In ECG signal processing, all the extensive anal ysis need the information of QRS positions as a basic [2]-[4]. QRS detectors have been regarded as a mature topic until the BSN is introduced, where, unfortunately, the ECG sensor requires real-time, miniature form factors and long lifetimes that push the limits of ultra low power circuit and system design.

Among the noises plaguing the ECG are the power-line interference, baseline drift, motion artifacts, electrode contact noise.

Much efforts have been given to the frequency-based ECG fil tering algorithms for QRS detection, i.e., a band-pass filter with a center frequency in the range of 10-17 Hz. After passing the filter, the signal may be squared or averaged over a number of samples to obtain the place of QRS waves. But these techniques suffer from the fact that frequency bands of the noise/other com addition, in order to accurately detect the QRS,

prior knowledge of frequency spectrums of different components in the recorded ECG signal is required through an additional training.

\section{Normal Ecg Waveform Signal}

These are representations of of electrical activity created by depolarization and repolarization of the atria and ventricles.If the electrical current is flowing towards the lead then a positive deflection will be seen. If flowing away from lead then a negative deflection will be seen. Wave forms that are above and below the Isoelectric line are called biphasic.

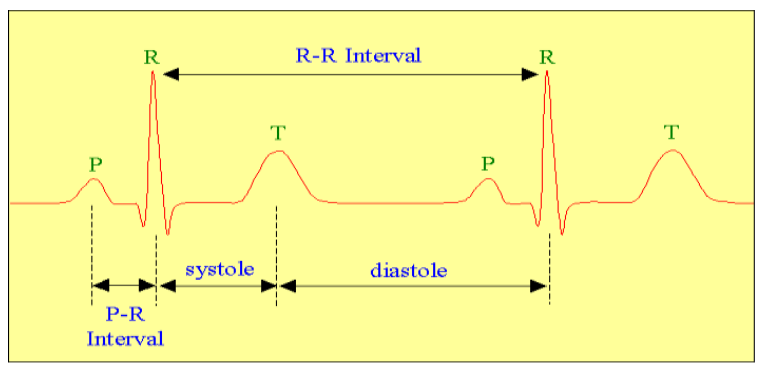

P Wave

Fig 1: ECG waveform

Electrical impulses originating in the SA node trigger atrial depolarization. The normal $\mathrm{P}$ wave is no more than 0.1 second in duration and $2.5 \mathrm{~mm}$ high. The direction of electrical activity is from SA to AV node. The $\mathrm{P}$ wave is a representation of the time it takes for atrial depolarization. It is viewed normally as small and curved with a positive deflection. Seen at it's tallest on lead II. 


\section{T Wave}

Ventricular repolarization which follows ventricular depolarization, is represented by the $\mathrm{T}$ wave. Its shape is rounded and taller and wider than the $\mathrm{P}$ wave. It is also more sensitive to physiologic and hormonal changes in shape but usually presents as a positive deflection $5-10 \mathrm{~mm}$ in height.

\section{U Wave}

After T wave an ECG can sometimes show a $\mathrm{U}$ Wave. It is of the same deflection as $\mathrm{T}$ Wave and similar to shape to $\mathrm{P}$ Wave. The $\mathrm{U}$ Wave is thought to represent late repolarization of the Purkinje fibers in the Ventricles and is more often not shown on a rhythm strip.

\section{Intervals and Segments}

Interval refers to the length of a wave plus the isoelectric line that follows it. The length of an interval ends when another wave begins. They are named by using the letters of both waves on either side. Intervals contain waves. Segments refers to the baseline between the end of one wave and the beginning of the next wave. Segments are the lines between waves. See diagram below.

\section{PR Interval}

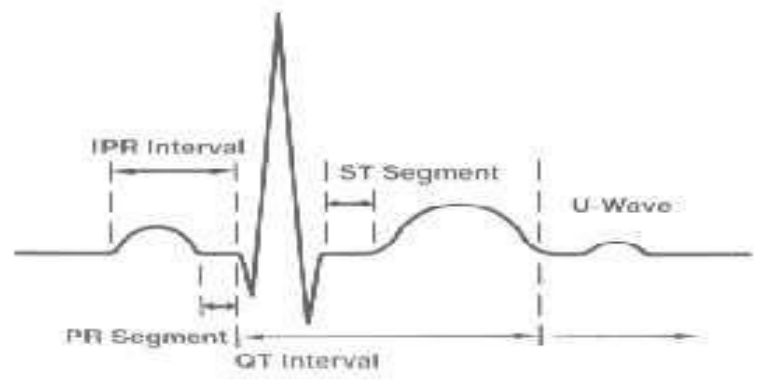

Fig .2: ECG waveform with intervals

Is the length along the baseline from the beginning of the $\mathrm{P}$ wave to the beginning of th QRS complex. This Is normally 0.12 to 2.0 seconds in duration.

\section{QT Interval}

Is the beginning of the QRS complex to the end of the T wave. In the presence of a $\mathrm{U}$ wave the measure should be from the beginning of the QRS complex to the end of the $U$ wave.

\section{ST Segment}

Is the length between the end of the $\mathrm{S}$ wave of the QRS complex and the beginning of the $\mathrm{T}$ wave. It is electrically neutral.

\section{PR Segment}

It represents the delay in conduction from atrial depolarization to the beginning of ventricular depolarization. It is also electrically neutral.

\section{The Complex}

The ECG has only one complex which is the QRS. it can be described as when one wave follows another without intervals, segments or isoelectric lines between them.

The QRS complex represents ventricular depolarization. it consists of three waveforms. The normal complex begins with a downward deflection known as the $\mathrm{Q}$ wave, followed by an upward deflection called the $\mathrm{R}$ wave. The next downward deflection will be the $\mathrm{S}$ wave. All ventricular complexes are known as QRS complexes even if every wave is not present in all complexes. The normal QRS is 0.04 to 0.10 seconds. You may have also been told .08 to .11 the importance is minimal.

Theoretical considerations

\section{Theory Of Mathematical Morphology}

The two principal morphological operations are dilation and erosion [1]. Dilation allows objects to expand, thus potentially filling in small holes and connecting disjoint objects. Erosion shrinks objects by etching away (eroding) their boundaries. These operations can be customized for an application by the proper selection of the structuring element, which determines exactly how the objects will be dilated or eroded.

Notations

Black pixel: in grayscale values for a 8 bits/pixel indexed image its value will be 0 
white pixel: in grayscale values for a 8 bits/pixel

The dilation

The dilation process is performed by laying the structuring element $\mathrm{B}$ on the image A and sliding it across the image in a manner similar to convolution (will be presented in a next laboratory). The difference is in the operation performed. It is best described in a sequence of steps:

1. If the origin of the structuring element coincides with a 'white' pixel in the image, there is no change; move to the next pixel.

2. If the origin of the structuring element coincides with a 'black' in the image, make black all pixels from the image covered by the structuring element.

Notation:

$A \oplus \mathrm{B}$

indexed image its value will be 255

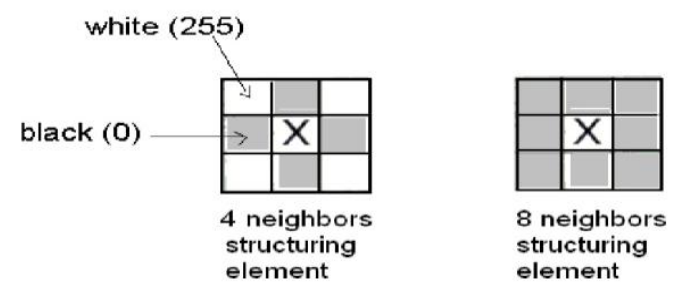

Fig 3:Typical shapes of the structuring elements (B)

The erosion

The erosion process is similar to dilation, but we turn pixels to 'white', not 'black'. As before, slide the structuring element across the image and then follow these steps:

1. If the origin of the structuring element coincides with a 'white' pixel in the image, there is no change; move to the next pixel.

2. If the origin of the structuring element coincides with a 'black' pixel in the image, and at least one of the 'black' pixels in the structuring element falls over a white pixel in the image, then change the 'black' pixel in the image (corresponding to the position on which the center of the structuring element falls) from 'black' to a 'white'.

Notation:

$A \Theta B$

\section{Opening and closing}

These two basic operations, dilation and erosion, can be combined into more complex sequences. The most useful of these for morphological filtering are called opening and closing. Opening consists of an erosion followed by a dilation and can be used to eliminate all pixels in regions that are too small to contain the structuring element. In this case the structuring element is often called a probe, because it is probing the image looking for small objects to filter out of the image. See Fig 4, for the illustration of the opening process.

Notation:

$A \circ B=(A \Theta B) \oplus B$

Closing consists of a dilation followed by erosion and can be used to fill in holes and small gaps. we see that the closing operation has the effect of filling in holes and closing gaps. Comparing the left and right images from Fig. 5, we see that the order of operation is important. Closing and opening will generate different results even though both consist of erosion and dilation.

Notation:

$$
A \bullet B=(A \oplus B) \Theta B
$$




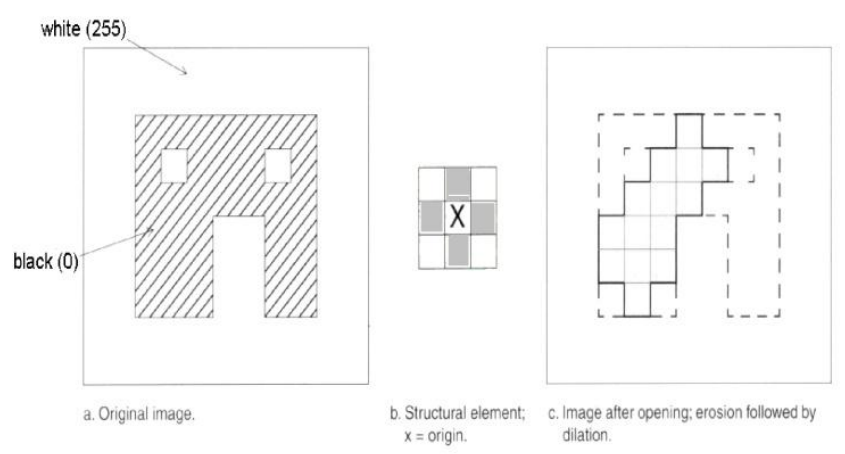

Fig 4:Illustration of the opening process

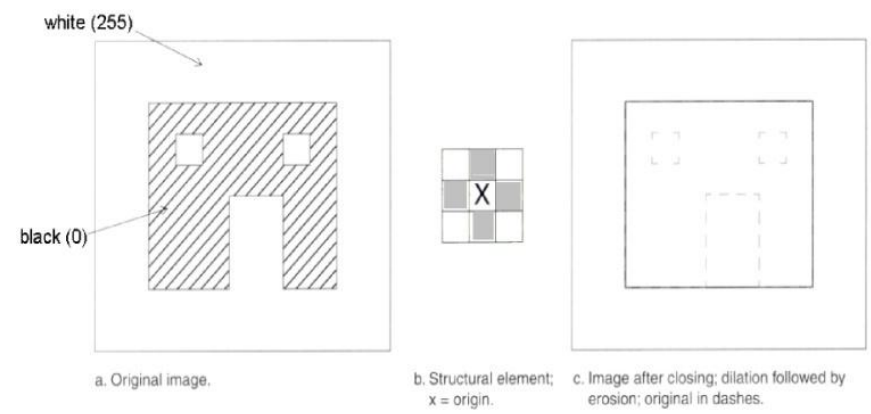

Fig 5:Illustration of the closing process

\section{Proposed Qrs Detection Algorithem}

Detection of QRS complexes equals to the distinguishing of a group of consecutive positive and negative peaks. As mentioned above, originating from 2-D image processing, mathe-matical morphological technology extracts the effective information based on shapes in the image, not pixel intensities like conventional methods. A fundamental advantage of mathemati- cal morphology applied to signal processing is that it is intuitive since it works directly on the spatial domain: the structuring elements considered as the "basic bricks" play the same role frequencies do in the analysis of the frequently used frequency filters. Another advantage is that it leads to better reproducible results because of the strong mathematical foundation. The simplicity in terms of computation and hardware implementation is also one of its main advantages.

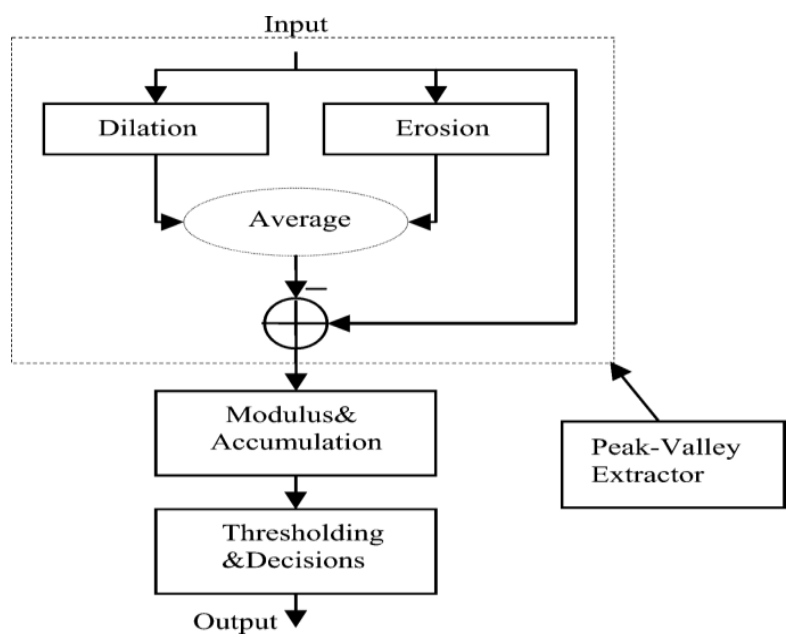

Fig. 6: Block diagram of proposed algorithm

The mathematical morphology was recently introduced into 1-D ECG processing [7]-[12], demonstrating the effectiveness of removal of impulsive noise and background normalization. However, existing [7]-[12] employed 8, 8, 8, 6, 4, and 2 high order opening/closing operators or their computational combinations to realize the QRS detectors, respectively. In order to further alleviate the implementation load and reduce the power consumption, a VLSI friendly QRS detector only based on the most fundamental dilation and erosion operator, as mentioned. 
As shown below, this algorithm will offer the lowestcost of hardware implementation in both power and size when compared to [7]-[12].

The diagram of the proposed algorithm is shown in Fig.6. The proposed morphology operator filtering plays the most critical role in the proposed algorithm which removes the noise and baseline drift and suppresses the P/T waves in ECG signal Then the multipixel modulus accumulation is used to enhance the QRS complex. Finally, the threshold is applied to decide the heart rate. The detailed discussions on each section in Fig. 6 are presented in the following subsections. The standard MIT/BIH ECG database [13] and our own wearable ECG data are used to demonstrate the superiority of proposed QRS detector

An adaptive threshold is used as the decision function in connection with the proposed transformation for QRS detection.

\section{Results}

The ECG signal obtained in the matlab and the processed ECG signal output is shown below. The pecentage of utiliztion of area in chip is also very less that we can see in modelsim.

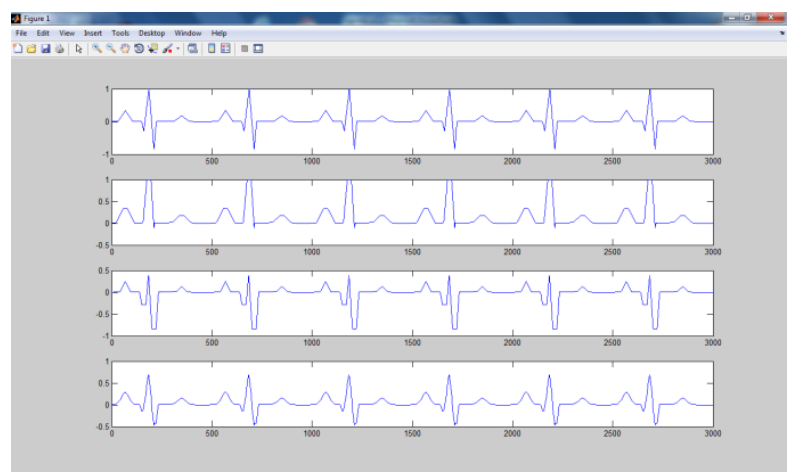

Fig 7: Reult of simulation in matlab

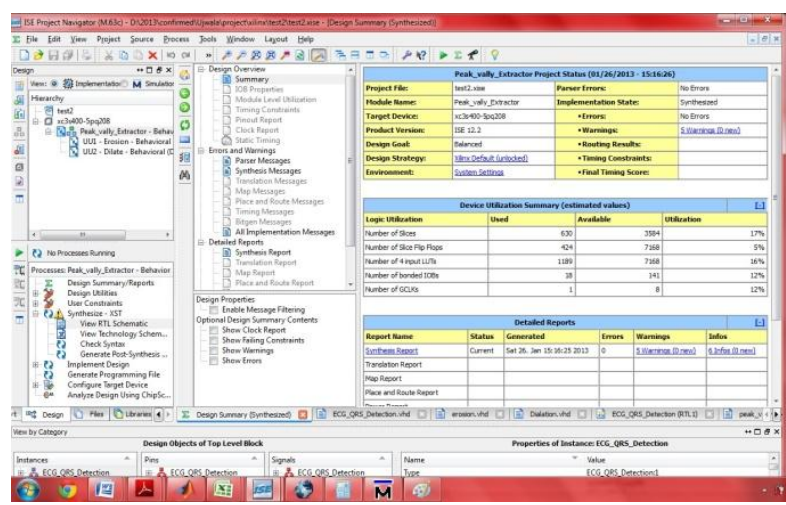

Fig 8: Description of design summary in modelsim

\section{References}

[1]. G. Yang, Body Sensor Networks. New York: Springer, 2006.

[2]. B. U. Kohler, C. Hennig, and R. Orglmeister, "The principles of soft- ware QRS detection," IEEE Eng. Med. Biol. Mag., vol. 21, no. 1, pp. 42-57, Jan. 2002.

[3]. F. Zhang and Y. Lian, "Novel QRS detection by CWT for ECG sensor," in Proc. Int. Conf. IEEE Biomed. Circuits Syst., Montreal, ON, Canada, Nov. 2007.

[4]. H. Lee and K. M. Buckley, "ECG data compression using cut and align beats approach and 2D transforms," IEEE Trans. Biomed. Eng., vol. 46, no. 5, pp. 556-564, May 1999.

[5]. A. Gasterators, I. Andreadis, and Ph. Tsalides, "Fuzzy soft mathemat- ical morphology," in IEEE Proc. Vis. Imag. Signal Process., Feb. 1998, vol. 145, pp. 41-49.

[6]. F. Zhang, C. Li, and L. Shi, "Detecting and tracking dim moving point target in IR image sequences," Infrared Phys. Technol., vol. 46, pp. 323-328, Apr. 2005.

[7]. F. Zhang and Y. Lian, "Effective ECG QRS detection based on multi- scale mathematical morphology filtering," IEEE Trans. Biomed. Cir- cuits Syst., vol. 3, no. 4, pp. 220-228, Aug. 2009.

[8]. J. Hu and S. Bao, “An approach to QRS complex detection based on multiscale mathematical morphology," in Proc. 3rd IEEE Int. Conf. Biomed. Eng. Inform. (IEEE BMEI 2010), 2010, p. 725.

[9]. C.-H. N. Chu and E. J. Delp, "Impulsive noise suppression and back- ground normalization of electrocardiogram signals using morpholog- ical operators," IEEE Trans. Biomed. Eng., vol. 36, no. 2, pp. 262-273, Feb. 1989.

[10]. P. E. Trahanias, “An approach to QRS complex detection using mathematical morphology," IEEE Trans. Biomed. Eng., vol. 40, no. 2, pp. 201-205, Feb. 1993. 
[11]. Y. L. Chen and H. L. Duan, "A QRS complex detection algorithm based on mathematical morphology and envelope," in Proc. 27th Annu. Int. Conf. IEEE EMBS, Shanghai, China, Sep. 2005, pp. 4654-4657.

[12]. F. Gritzali, “Towards a generalized scheme for QRS detection in ECG waveforms," Signal Process., vol. 15, pp. 183-192, Sep. 1988

[13]. T. Hoang, J. Son, Y. Kang, C. Kim, H. Chuang, and S. Kim, "A low complexity, low power, programmable QRS detector based on wavelet transform for implantable pacemaker IC," in IEEE Int. SOC Conf., Sep. 24-27, 2006, pp. 160-163.

[14]. A. Ruha and S. Sallinen, "A real-time microprocessor QRS detector system with a 1-ms timing accuracy for the measurement of ambulatory HRV," IEEE Trans. Biomed. Eng., vol. 44, no. 2, pp. 159-167, Feb. 1997.

[15]. J. Pan and W. J. Tompkins, “A real time QRS detection algorithm," IEEE Trans. Biomed. Eng., vol. 32, no. 3, pp. 230-236, Mar. 1985.

[16]. Q. Xue, Y. H. Hu, and W. J. Tompkins, "Neural-network-based adap- tive filtering for QRS detection,” IEEE Trans. Biomed. Eng., vol. 39, no. 4, pp. 315-329, Apr. 1992. 